\title{
Patterned turbulence and relaminarization in MHD pipe and duct flows
}

\author{
Dmitry Krasnov ${ }^{1}$, Oleg Zikanov ${ }^{2}$, and Thomas Boeck ${ }^{1, *}$ \\ ${ }^{1}$ Institute of Thermodynamics and Fluid Mechanics, Ilmenau University of Technology, 98684 Ilmenau, Germany \\ ${ }^{2}$ Mechanical Engineering, University of Michigan - Dearborn, USA
}

\begin{abstract}
We present results of a numerical analysis of relaminarization processes in MHD duct and pipe flows. It is motivated by Julius Hartmann's classical experiments on flows of mercury in pipes and ducts under the influence of magnetic fields. The simulations, conducted both in periodic and non-periodic settings, provide a first detailed view of flow structures that have not been experimentally accessible. The main novelty of the analysis is very long (tens to hundreds of hydraulic diameters) computational domains that allows to discover new flow regimes with localized turbulent spots near the side walls parallel to the magnetic field. The computed critical parameters for transition as well as the friction coefficients are in good agreement with Hartmann's data.
\end{abstract}

(c) 2014 Wiley-VCH Verlag GmbH \& Co. KGaA, Weinheim

The processes of flow re-laminarization in tubes (i.e. pipes and ducts) were the first MHD phenomena studied experimentally [1]. The experiments were performed for flows in pipes and ducts of different aspect ratios, subjected to a uniform transverse magnetic field. The flows had moderate values of the Reynolds number $R e=2000 \ldots 5000$ and low-to-moderate magnetic fields $\boldsymbol{B}$. Laminarization was detected by measuring the pressure drop in the test section and comparing these values with theoretical ones for laminar MHD channel flow. It was found that complete flow laminarization occurs in a range of $R \approx 200 \ldots 220$, where $R$ is the "local" Reynolds number based on the Hartmann layer thickness $\delta_{H a}$.

Since then the transition in MHD pipe and duct flows is usually associated with certain, "critical", value of the Reynolds number $R$. This particular role of $R$ as a control parameter has been confirmed in various experimental studies. On the other hand, theoretical works have not been able to find a plausible explanation of transition in the MHD tube flows so far. One reason for this is the non-trivial structure of the laminar velocity distribution in tube flows, which is characterized by a flat core and thin electromagnetic boundary layers: (i) the Hartmann layers at the walls perpendicular to the magnetic field and (ii) the side layers, called either Shercliff layers (in rectangular ducts) or Roberts layers (in circular pipes), on the walls parallel to the field. These layers have different nature and, correspondingly, different thickness. For laminar duct flow the Hartmann layers have a typical thickness of $\delta_{H a} \sim 1 / B$, whereas the side layers scale as $\delta_{S h} \sim 1 / B^{1 / 2}$ and are thicker than the Hartmann layers. Thus the shear, although of different strength, is concentrated in Hartmann and side layers, so that both of these two regions may support instabilities leading to turbulence. The role of $R$ as decisive parameter for transition points to the Hartmann layers as origin of transition. This point may receive much favour, as the shear and velocity gradients are stronger in the Hartmann layers. However, there are indications that instability first develops in the side layers, e.g. by an analysis of optimal linear perturbations in MHD duct flow [2]. In this work we summarize the results of our prior studies [3,4], where the experiments of Hartmann \& Lazarus [1] on flow relaminarization have been reproduced numerically using fully non-linear DNS. We also report first results on extending this analysis towards higher Reynolds numbers and stronger fields.

We consider the flow of an incompressible, electrically conducting fluid (e.g. liquid metals) in rectangular duct or circular pipe subjected to a uniform magnetic field $\boldsymbol{B}$. MHD effects are considered in the limit of small magnetic Reynolds number, i.e. the quasi-static approximation [5] is applied. The governing non-dimensional equations and boundary conditions are given in [3]. The non-dimensional parameters are the Reynolds number $\operatorname{Re} \equiv U_{q} a / \nu$ and the magnetic interaction parameter $N \equiv H a^{2} / R e$, where $H a \equiv B a(\sigma / \rho \nu)^{1 / 2}$ is the Hartmann number. Here $U_{q}$ is the mean flux velocity, $a$ is the half-diameter (pipe) or half-height (duct) and $\sigma$ is the electrical conductivity. We also notice that the "local" Reynolds number $R$, mentioned above, can be defined as $R=U_{q} \delta_{H a} / \nu=R e / H a$. The governing equations are solved numerically by our in-house DNS solvers, implemented for rectangular (duct) and cylinder (pipe) geometries. The solvers are based on finite-difference method described in [6]. The spatial discretization of $2^{\text {nd }}$ order is on a non-uniform structured grid formed along the lines of the Cartesian (duct) or cylindrical (pipe) coordinate system. The time integration uses projection-correction procedure to satisfy incompressibility. The computational grid can be clustered in the wall-normal (or radial) directions to resolve thin MHD boundary layers.

The simulations have been conducted at $R e=3000$ and 5000 and $H a=0 \ldots 30$ for two different settings: flows periodic in the streamwise $x$-direction and flows with non-periodic inlet/exit conditions. Periodic conditions correspond to a fully developed flow under perfectly uniform magnetic field, whereas the non-periodic formulation is more realistic and allows us to mimic the experimental flow conditions with entry and exit effects at the test section. Turbulent inflow conditions for non-periodic simulation are supplied from a periodic DNS running at the same $R e$ and grid spacing. To capture the evolution of turbulent spots, we have used long computational domains, up to $L_{x}=128 \pi$ in terms of the hydraulic radius $a$.

\footnotetext{
* Corresponding author: Email Thomas.Boeck@tu-ilmenau.de
} 
We start our discussion with comparison of integral quantities. The only available parameter from the H. \& L. study [1] was the pressure drop between two manometers, which can be recalculated in the friction coefficient $f=2 a d p / d x /\left(\rho U^{2}\right)$. Fig. 1 shows that the friction coefficient $f$ at $R e=3000$ agrees well between experiments and both periodic and nonperiodic simulations. Initially, the friction is reduced because of the magnetic damping of turbulence, then, for $\mathrm{Ha}>15$ it increases linearly with $\mathrm{Ha}$. At this point the laminar Hartmann layers provide the dominant contribution to the total friction. Relaminarization occurs close to the minimum of the friction coefficient, which corresponds to a parameter $R \approx 200$.

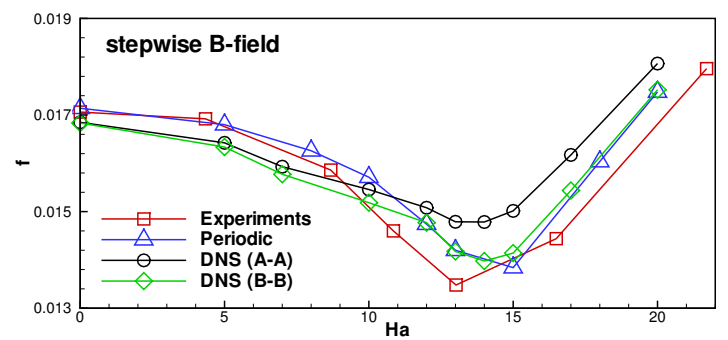

Fig. 1: Friction coefficient vs. Hartmann number $\mathrm{Ha}$ shown for the results of simulations and experimental data [1].

Another aspect of our study is the appearence of patterned turbulence in MHD tubes. The phenomenon of patterned turbulence, i.e. coexistence of laminar and turbulent zones, is known for hydrodynamic wall-bounded shear flows (such as puffs and slugs in pipe flow $[7,8])$, however, it has not been clearly demonstrated for MHD tube flows. In the present study the patterned turbulence has been identified for MHD pipes and ducts in both periodic and nonperiodic settings. In all our simulations these regimes appear in the transitional range of $H a$ numbers, corresponding to $R \approx 220 \ldots 200$, i.e. at the edge of laminarization as suggested by the dependence of friction coefficient $f$ on $\mathrm{Ha}$.

The regimes with patterned turbulence are illustrated in figure 2 for non-periodic flow simulations at $R e=3000$ and $H a=13,14,15$. Here, the core region and Hartmann boundary layers remain laminar, whereas the turbulent spots, or puffs, are tightly localized in the sidewall layers. The puffs tend to form staggered patterns, as seen at $H a=13$, although the specific arrangement is largely influenced by either initial or inlet flow conditions. At $H a=14$ the pattern with visually weaker spots still develops. Finally, at $\mathrm{Ha}=15$ the flow becomes essentially laminar at $x \approx 100$, although sporadic events can appear, but these spots die out quickly. For stable puff-patterns one can identify a characteristic length of a single spot $L_{\text {puff }} \approx 130-150$ in terms of the sidewall layer thickness $\delta_{S h}$. We have also analyzed the temporal evolution of the puffs and identified multiple events as, e.g., merging and splitting of two or more neighboring puffs or two opposite-side spots forming a 'locked' state and traveling together.

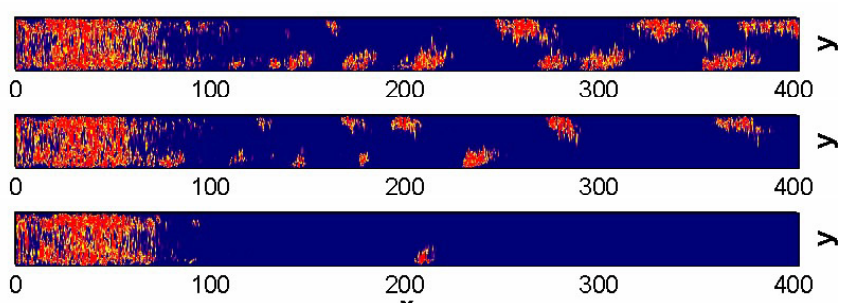

Fig. 2: Turbulent kinetic energy in the $(x, y)$-plane at $z=0$ (the midplane perpendicular to the magnetic field) at $\mathrm{Re}=3000$ and $\mathrm{Ha}=$ $13,14,15$ (from top to bottom). The results correspond to the case of stepwise magnetic field beginning at $x \approx 46$.

In this study numerical simulations of MHD duct and pipe flows have been performed to reproduce the classical experiments by Hartmann \& Lazarus [1]. One distinct features that we have identified is the co-existence of laminar and turbulent regions at the edge of laminarization. The peculiarity of the MHD flows is the localization of these turbulent zones in the sidewall layers. The exact re-laminarization threshold cannot be identified in a clear-cut way, because the specific flow state - either fluctuating or fully laminar - strongly depends on the initial and/or inlet flow conditions. Instead, one can talk about a range of Reynolds numbers $\mathrm{R}=\mathrm{Re} / \mathrm{Ha}$ where the change of flow regime is expected. In our simulations this transitional range is found at $R \sim 200 \ldots 220$, which is in very good agreement with experiments. The friction coefficient $f$ also agrees with the experiments. It is, however, important to notice that parameter $f$ provides no indication of the existence of turbulent zones as their impact on the total friction is very low. This conclusion is particularly well supported by our preliminary simulations performed at $R e=10^{5}$ and $\mathrm{Ha}=450 \ldots 500$ [9]. In this "high-parameter" range the feature of localized turbulent spots is perfectly revealed too. The puffs are stretched along the magnetic field direction and resemble objects known as "turbulent bands". They become very thin and occupy the entire sidewall layer. We conclude that further work is necessary to resolve the details of transition in the side layers, especially at strong fields, and to explain why the parameter $R$ determines the transition in a broad range of $R e$ and Ha numbers.

Acknowledgements TB and DK acknowledge financial support from the Helmholtz Alliance "Liquid Metal Technologies" and the Deutsche Forschungsgemeinschaft. OZ acknowledges financial support from the U.S. National Science Foundation (Grants CBET 0966557 and CBET 1232851). Computer resources were provided by the computing centers of Forschungszentrum Jülich (NIC) and TU Ilmenau.

\section{References}

[1] J. Hartmann and F. Lazarus, K. Dan. Vidensk. Selsk. Mat. Fys. Medd. 15(7), 1-45 (1937).

[2] D. Krasnov, O. Zikanov, M. Rossi, and T. Boeck, J. Fluid Mech. 653, 273-299 (2010).

[3] D. Krasnov, A. Thess, T. Boeck, Y. Zhao, and O. Zikanov, Phys. Rev. Lett. 110, 084501 (2013).

[4] O. Zikanov, D. Krasnov, Y. Li, T. Boeck, and A. Thess, Theor. Comp. Fluid Dyn. 28, 319-334 (2014).

[5] P. A. Davidson, An Introduction to Magnetohydrodynamics (Cambridge University Press, 2001).

[6] D. Krasnov, O. Zikanov, and T. Boeck, Comp. Fluids 50, 46-59 (2011).

[7] O. Reynolds, Philos. T. R. Soc. A 174, 935-982 (1883).

[8] I. J. Wygnanski and F. H. Champagne, J. Fluid Mech. 59, 281-335 (1973).

[9] O. Zikanov, D. Krasnov, T. Boeck, A. Thess, and M. Rossi, Applied Mechanics Reviews 66, 030802-1 (2014). 